\title{
Blood will out: vascular contributions to Alzheimer's disease
}

\author{
Sidney Strickland \\ Patricia and John Rosenwald Laboratory of Neurobiology and Cenetics, The Rockefeller University, New York, New York, USA.
}

\begin{abstract}
The fundamental pathology in Alzheimer's disease $(A D)$ is neuronal dysfunction leading to cognitive impairment. The amyloid- $\beta$ peptide $(A \beta)$, derived from amyloid precursor protein, is one driver of $A D$, but how it leads to neuronal dysfunction is not established. In this Review, I discuss the complexity of $A D$ and possible cause-and-effect relationships between $A \beta$ and the vascular and hemostatic systems. $A D$ can be considered a multifactorial syndrome with various contributing pathological mechanisms. Therefore, as is routinely done with cancer, it will be important to classify patients with respect to their disease signature so that specific pathologies, including vascular pathways, can be therapeutically targeted.
\end{abstract}

\section{Introduction}

Alzheimer's disease $(\mathrm{AD})$ is the most prevalent form of dementia, affecting approximately 5.5 million Americans. With an aging population worldwide, it is predicted to be a public health crisis in the coming decades. In spite of extensive preclinical and clinical efforts, little progress has been made in preventing or reversing this disease. A better understanding of $\mathrm{AD}$ requires reconceptualizing the disorder as an amalgam of dysfunctions rather than one pathology. An analogous evolution has occurred in cancer biology; decades ago, the search for a "magic bullet" for cancer treatment suggested that cancer was one disease and that it would be possible to identify a single drug to cure all forms. With today's advanced knowledge of the many mechanisms that lead to cancer, that original concept is very dated. Yet, researchers today still discuss "a" cure for AD, implying that a single pathological pathway is responsible for all cases. For example, the tag line on a 2016 cover of Time magazine read, "The Alzheimer's Pill."

It is becoming obvious that $\mathrm{AD}$, like cancer, is a complex disease with multiple pathogenic mechanisms. One pathway that has been largely overlooked is vascular dysregulation. For example, a relatively recent and highly cited hypothetical temporal ordering of $\mathrm{AD}$ pathologies did not include vascular pathology in the possible contributors to the disease (1). Accumulating evidence, detailed in this review, indicates that vascular dysregulation plays a major role in cognitive decline. Undoubtedly, attacking the disease successfully will involve identifying and targeting various mechanisms, as has been done in the revolution in cancer treatment.

\section{Multiple mechanisms can contribute to AD pathology}

In cancer, the fundamental pathology is unregulated cell division. However, this aspect by itself is not necessarily lethal. Other mechanisms - for example, immune system evasion and metastasis - can compound too much cell division and exacer-

Conflict of interest: The author has declared that no conflict of interest exists. Reference information: / Clin Invest. 2018;128(2):556-563.

https://doi.org/10.1172/JCI97509. bate the morbidity of the disease. Cancer treatments have been developed to counteract these ancillary pathways, and these treatments can improve patient outcome. One can envisage a similar situation for AD. The fundamental pathology is neuronal dysfunction causing cognitive decline, and this pathway can be accelerated by many other abnormalities such as defects in autophagy (2), synaptic toxicity (3), and oxidative stress/ mitochondrial dysfunction (4). Of all the many possible contributors, inflammation (5) and vascular abnormalities $(6,7)$ appear to be especially significant (Figure 1 and see below).

\section{Could vascular mechanisms benefit AD diagnosis or treatment?}

If ancillary mechanisms can contribute to $\mathrm{AD}$, one consideration is how to determine the relevance of these pathways in individual patients, especially if the mechanisms are not specific to AD. Again, cancer provides a useful analogy. Estrogen and progesterone receptor expression is not specific to breast cancer, and also occurs in normal tissues and other cancers. Nevertheless, in patients with breast cancer, hormone receptor expression predicts response to antiestrogen therapy such as tamoxifen and such therapy improves patient outcome. Likewise, patients with cognitive impairment could be tested for vascular abnormalities and inflammation. Those that exhibit these ancillary pathologies could then be treated for those conditions. As with cancer, it seems likely that this approach would benefit this subset of patients.

\section{Is there a connection between vascular pathology and $A D$ ?}

The classical pathological hallmarks of $\mathrm{AD}$ are amyloid- $\beta$ peptide $(\mathrm{A} \beta)$ plaques, tau tangles, neuroinflammation, and neuronal loss (8). The $A \beta$ deposition can be in the brain parenchyma or in and around cerebral blood vessels, a condition known as cerebral amyloid angiopathy (CAA), discussed below (9). Less discussed is that $\mathrm{AD}$ is very often associated with cerebrovascular abnormalities $(6,10-13)$. These cerebral pathologies include microinfarcts, hemorrhage, decreased cerebral blood flow, small vessel disease, and white matter abnormalities $(14,15)$. Three recent studies of 


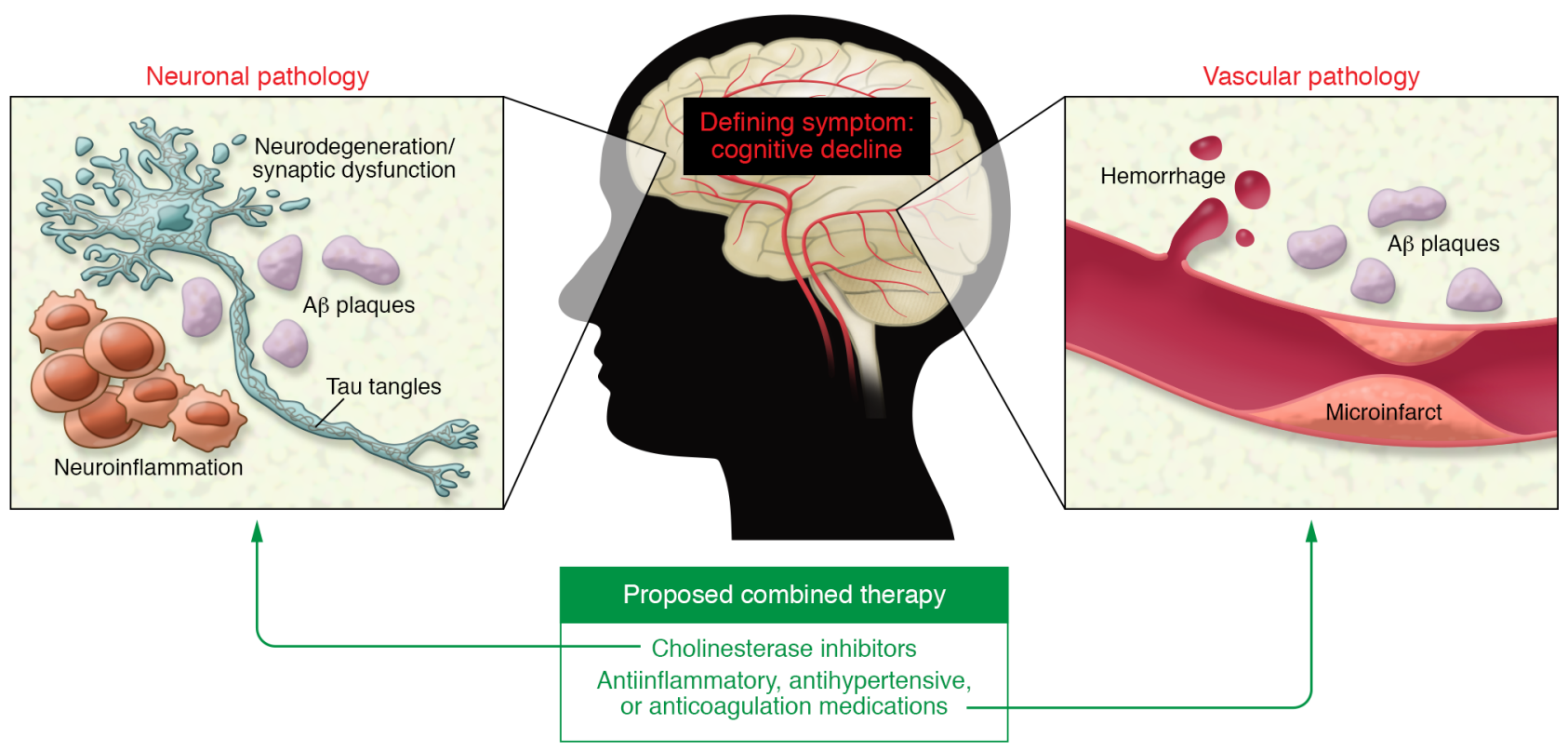

Figure 1. Multiple pathogenic pathways contribute to and provide therapeutic targets for AD. While neuronal dysfunction is the defining pathology underlying cognitive decline in AD, this disease is likely the product of multiple pathogenic mechanisms and might benefit from combination therapy (128130). Increasing evidence implicates vascular dysfunction in AD pathogenesis. A $\beta$ plaques and tau tangles drive neuronal dysfunction and neuroinflammation through mechanisms that are not fully established. $A \beta$ is also implicated in vascular pathology, having been shown to interact with fibrin(ogen) and the contact system. The prevalence of vascular dysfunction in patients exhibiting cognitive decline suggests that combining existing treatment strategies targeting neuronal dysfunction (e.g., cholinesterase inhibitors) and vascular dysfunction (e.g., antiinflammatory, antihypertensive, or anticoagulant medications) may advance $A D$ treatment efficacy.

vascular disease in AD autopsy samples showed that concurrent vascular disease is very common in $\mathrm{AD}$ and strongly correlates with cognitive dysfunction (16-18).

Reinforcing the concept that the vascular system influences $\mathrm{AD}$, multiple studies have reported that exercise, which improves cerebrovascular health, can decrease the risk and/or delay progression of dementia. Benefits of increased physical activity include improved memory performance and reduced hippocampal atrophy (19-21), increased gray matter volume and production of neurotrophic factors (22), lower risk of mortality $(23,24)$, and reduced risk of $\mathrm{AD}(25)$. However, this association is complex (26), as exercise could also have nonvascular benefits, and other studies have found no improvement in risk of dementia with exercise (27). Mechanisms by which exercise could influence $\mathrm{AD}$ are discussed below.

Another link between AD and the vascular system is CAA, the deposition of $A \beta$ in and around blood vessels of the brain (9), which affects $80 \%$ to $95 \%$ of $\mathrm{AD}$ patients $(15,16)$. CAA can cause blood vessel occlusion, microinfarcts, ischemia, microbleeds, and inflammation, conditions that can weaken the blood vessel wall and cause life-threatening hemorrhage $(8,15,28)$. All of these conditions could contribute to neuronal death that is associated with $\mathrm{AD}$ progression.

\section{$A D$ and vascular dysfunction: independent or causal pathologies?}

It is clear that vascular dysfunction can itself lead to cognitive decline. However, the coexistence of $\mathrm{AD}$ and cerebrovascular pathology prompts questions of whether these conditions are always independent comorbidities, as they are both more prevalent in the aged population. If so, there could be synergistic effects on brain function. Alternatively, there could be a mechanistic link between $\mathrm{AD}$ and vascular pathology $(29,30)$.

\section{Early vascular pathologies in late-onset AD patients}

A recent study used a multifactorial, data-driven analysis to examine various pathologies in 1,171 healthy and late-onset AD (LOAD) subjects and assign a temporal ordering of disease progression (31). The conclusion of this study was that vascular dysregulation, measured by arterial spin labeling of cerebral blood flow (CBF), is the earliest and strongest brain pathology associated with LOAD. A tentative ordering of abnormalities was (a) initial vascular dysregulation, (b) A $\beta$ deposition, (c) metabolic dysfunction, (d) functional impairment, and (e) gray matter atrophy. Given thresholds for detection, the exact order of the pathologies is not certain, but these analyses strongly "suggest that intra-brain vascular dysregulation is an early pathological event during disease development” (31).

\section{Vascular pathologies appear early in early-onset AD patients}

An analysis of early-onset AD (EOAD) patients who harbor autosomal-dominant mutations provides strong evidence for a connection between AD and vascular pathology (32). EOADassociated mutations occur in amyloid precursor protein (APP), from which the $A \beta$ peptide is derived, or in $\gamma$-secretase, which helps excise A $\beta$ from APP (33). These mutations are virtually fully penetrant, and by analyzing DNA sequences, one can iden- 


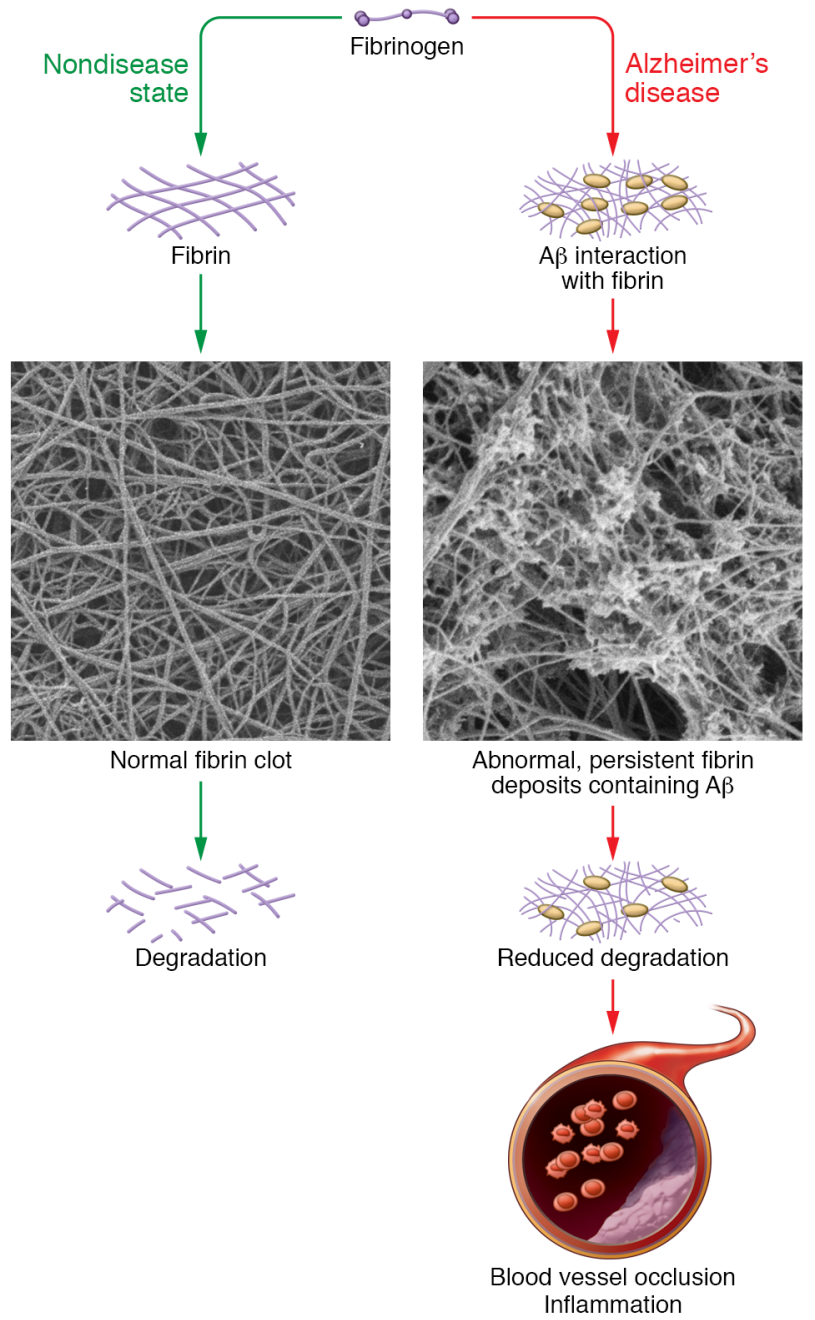

Figure 2. Possible influence of $A \beta$ on fibrin deposition and $A D$ pathology. The interaction of $A \beta$ with fibrinogen leads to increased formation of structurally abnormal fibrin clots that are resistant to degradation. This persistent fibrin and resulting predisposition towards blood vessel occlusion and inflammation could contribute to the neurodegeneration observed in AD. Images reproduced with permission from Cortes-Canteli et al. (53).

tify at the time of birth which patients will develop EOAD at a predictable age decades later. A comparison of mutation carriers and noncarriers reveals that the first abnormalities detected in these EOAD patients are alterations in $A \beta$ levels, which occur approximately 30 years before the onset of symptoms (estimated year of onset, EYO). The next pathology to emerge is white matter hyperintensities (WMHs), which are abnormalities observed during brain imaging. In carrier brains, WMH abnormalities were evident in general 6.6 years before EYO and in the occipital lobes as early as 22 years before EYO (32). This study did not measure CBF, so it cannot be compared to the results for LOAD patients discussed above.

WMHs are part of the spectrum of small vessel cerebrovascular disease $(34,35)$, including ischemic and hemorrhagic stroke, microbleeds, brain atrophy (36), chronic hypoperfusion (37), and an increase in blood-brain barrier (BBB) permeability, which causes fluid to leak into the surrounding brain tissue (38). What is notable is that these lesions predict the clinical outcome of $\mathrm{AD}$ as well as or more reliably than established markers such as hippocampal volume and atrophy $(39,40)$. In the EOAD study $(32)$, mutation carriers and noncarriers were relatively young, virtually identical demographically, and at equal risk for inheriting an autosomaldominant mutation. Thus, these findings provide strong evidence that vascular dysfunction does not reflect comorbidity or an independent pathophysiology, but rather that vascular dysfunction and $\mathrm{A} \beta$ pathology are causally related (32). In recent years, there has been evidence that WMHs are also associated with LOAD (41-43). In particular, WMH volume and amyloid pathology are linked in LOAD (42-44). However, it should be noted that one study found no association between WMHs and AD pathology (45).

Another link between $\mathrm{AD}$ and cerebrovascular disease stems from pathological outcomes in patients with autosomal-dominant mutations. Mutations in the $A \beta$ sequence at residues 21-23 (for example, Flemish, Dutch, Iowa, and Italian mutations) are associated with massive CAA, leading to weakening of the vessel wall and frequent infarcts and hemorrhage $(46,47)$. Mutations in presenilin-1, a protein component of $\gamma$-secretase that helps release A $\beta$ from APP, can also show severe cerebrovascular effects (48). All of this evidence indicates a connection between vascular pathology and AD.

\section{Mechanistic links between AD and vascular pathology}

$A \beta$ interaction with fibrin. Fibrin is the major protein component of blood clots and is critical for normal hemostasis (49). It is well established that in addition to its beneficial functions, persistent fibrin can lead to or exacerbate many pathological conditions, including atherosclerosis, rheumatoid arthritis, stroke, spinal cord injury, multiple sclerosis, muscular dystrophy, peripheral nerve regeneration, and even bacterial infection (50-52).

Postmortem AD patient brains have been analyzed with antibodies that do not distinguish between soluble fibrinogen and insoluble fibrin. These studies showed extensive fibrinogen/ fibrin deposition in the brain (53-60), which in some cases was attributed to a leaky BBB. Further studies using extraction procedures that remove all soluble fibrinogen and staining with fibrin-specific antibodies showed that insoluble fibrin is greatly increased in $\mathrm{AD}$ patient brains compared with nondemented controls, with differences in some regions reaching 100-fold (61). The fibrin deposition often colocalized with parenchymal $A \beta$ deposits and lysosome-associated membrane protein 1 (LAMP-1), which is upregulated in the human AD brain (62) and cerebrospinal fluid (CSF) (63) and enriched in dystrophic areas surrounding amyloid plaques in $\mathrm{AD}$ mice $(64,65)$ and human $\mathrm{AD}$ patient brains $(62,66)$. Fibrin is also found codeposited with $A \beta$ in areas of CAA $(53,67)$.

High levels of fibrinogen in plasma increase the risk for dementia $(68,69)$, and fibrinogen in CSF (70-72) and plasma (73, 74) can serve as a useful biomarker to identify $A D$ progression. In addition, fibrinogen has been proposed to be one of the few bloodbased biomarkers that is specific for $\mathrm{AD}$ and does not apply to other brain disorders (75).

The association of brain fibrin and $\mathrm{AD}$ prompts the question of whether this deposition is a result of $\mathrm{AD}$, or whether it contributes to the pathology. AD has been linked to the APP-derived $A \beta$ peptide, a known driver of $\mathrm{AD}$ pathology (76-78). $\mathrm{A} \beta$ binds to fibrinogen and fibrin $(79,80)$, leading to blood clots that are structurally 


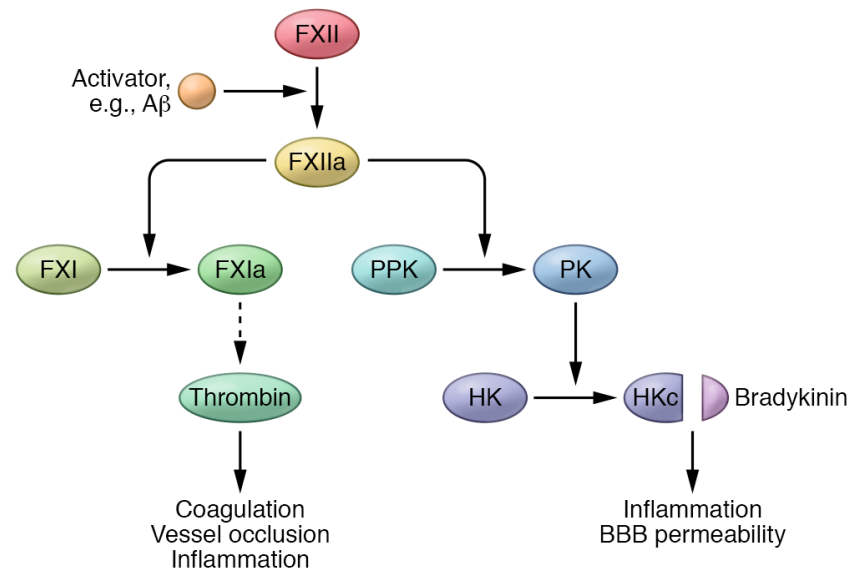

Figure 3. The contact activation system. $A \beta$-mediated dysregulation or overactivation of the contact system could contribute to the coagulopathy and inflammation observed in AD. A $\beta$ can trigger activation of factor XII (FXII) into FXIla. FXIla's activation of FXI initiates the intrinsic coagulation system and fibrin formation, whereas its activation of plasma prekallikrein leads to inflammation. PPK, plasma prekallikrein; PK, plasma kallikrein; HK, high molecular weight kininogen; HKc, cleaved HK. a = activated form.

abnormal and harder to degrade in vitro and in vivo than normal clots $(53,80)$. Thus, fibrin clots formed in $\mathrm{AD}$ patients and mice might be persistent and cause vessel occlusion and neuroinflammation, which could contribute to neuronal death and other disease pathologies (Figure 2).

Data in humans and mice indicate that fibrin deposition in $\mathrm{AD}$ brains is not simply a comorbidity due to the aging population at risk, but is instead a driving factor of the disease. Studies in atrial fibrillation patients with and without warfarin treatment showed that anticoagulants can protect against dementia (81). Earlier work with dementia patients reached the same conclusion (82). In $\mathrm{AD}$ mice, anticoagulant treatment is protective (83-85) and injection of fibrinogen into the brain induces demyelination (86), reminiscent of the white matter abnormalities observed in $\mathrm{AD}$ patients discussed above. More specifically, in AD mice treatment with a small molecule that blocks the $A \beta$-fibrin(ogen) interaction significantly improves the course of disease (87) and reducing fibrinogen levels results in reduced pathology and better cognitive ability (53, $60,61)$. Both of these studies substantiate a role for fibrin.

The mechanism by which fibrin accelerates neuronal degeneration remains unknown. There are two likely possibilities: (a) occlusion - fibrin clots are deposited in the vascular and perivascular space, resulting in reduced blood flow, increased $A \beta$ accumulation due to binding to clots, and neuronal damage due to deprivation of oxygen and nutrients; and (b) inflammation fibrin deposits drive a chronic inflammatory state that leads to cellular damage (50).

$\mathrm{AD}$ is associated with inflammation, which in some cases can be a beneficial response but in other cases can be toxic to cells $(88,89)$. AD patient brains have increased inflammation, mutations in immune-related molecules lead to an increased risk of $\mathrm{AD}$, and nonsteroidal antiinflammatory drugs show some effects at reducing $\mathrm{AD}$ risk (5). Furthermore, AD mice that are incapable of mounting a proinflammatory response show improvement in pathology and cognition $(90,91)$. Since fibrin is a driver of inflam- mation, it could therefore contribute to the initiation of the inflammation observed in $\mathrm{AD}$ (88).

The contact system, intrinsic blood coagulation, and $A \beta$. The contact system is initiated by activation of the serine protease factor XII (FXII). Once converted to its active serine protease form, FXIIa can launch both prothrombotic and proinflammatory pathways (92). Regarding the prothrombotic arm, FXIIa can activate factor XI (FXI), which leads to thrombin generation and fibrin formation via the intrinsic blood coagulation pathway (93). In the proinflammatory arm, FXII can activate plasma prekallikrein (PPK), which leads to the release of bradykinin via cleavage of high molecular weight kininogen (HK). Bradykinin is a potent nonapeptide vasodilator that can activate inflammatory processes (Figure 3 and ref. 94). Thus, the contact system can initiate vascular pathology and inflammation $(92,95)$, both of which have been implicated in $\mathrm{AD}$, and could contribute to disease pathology.

Many connections between FXII and AD have been reported, suggesting that this system may play a role in disease development. A $\beta$ plaques contain FXII (96), the AD brain parenchyma exhibits higher plasma kallikrein (PK) activity (97), and $\mathrm{AD}$ patients have increased HK cleavage in their CSF (98). Consistent with a role for the contact system, $\mathrm{AD}$ patients have higher plasma levels of FXIIa and increased HK cleavage compared with nondemented individuals (99). Experiments with a mouse model of AD also show increased contact system activation $(99,100)$.

The contact system can be triggered by $A \beta$, which can activate FXII (101-104). This increase in FXIIa leads to elevated thrombin generation, kallikrein activity, and HK cleavage in AD patient plasma (100). Increased contact system activation is also observed in $\mathrm{AD}$ mouse model plasma and in plasma from wild-type mice injected intravenously with $\mathrm{A} \beta 42$. These results demonstrate that A 442 -mediated contact system activation can occur in the $\mathrm{AD}$ circulation and suggest new pathogenic mechanisms, diagnostic tests, and therapies for AD.

Experiments in mice suggest that the contact system is a direct effector of AD pathology. Depletion of FXII using antisense oligonucleotide treatment ameliorates pathology in $\mathrm{AD}$ mice in earlystage disease (100). These results indicate that dysregulated contact system activation contributes to $\mathrm{AD}$ pathology.

Contact system activation could be used in conjunction with other diagnostic procedures such as PET and MRI imaging, CSF analysis, and/or cognitive testing to help stratify patients by their pathological profile and help guide therapy. Furthermore, a link between FXII activation and the pathogenesis of $\mathrm{AD}$ provides a possible novel approach to treatment since the contact system is an attractive target for therapy (105). Humans deficient in FXII and mice with knockout of the FXII, FXI, or Kng1 (encoding HK) gene all have normal hemostasis (95). However, deficiencies in the contact system protect mice from clotting after arterial injury and experimental cerebral ischemia $(92,106)$.

Based on these considerations, a promising therapeutic approach to slow disease progression without affecting normal hemostasis would be to block activation of the contact system. This approach would also block bradykinin release from HK and reduce inflammation. Thus, the contact system may reveal new targets to suppress both thrombotic and inflammatory contribu- 
tions to AD progression. Positive results could be applied to AD patients rapidly; a small molecule inhibitor of PK, ecallantide, is already FDA approved for treatment of hereditary angioedema, a condition that results from excess contact system activation (107). Furthermore, an antibody inhibitor of PK (108) is slated for a phase 3 trial and possible FDA approval by 2018. Some of these reagents might be useful for the treatment of $\mathrm{AD}$ in the future.

\section{Could vascular pathology trigger AD?}

The above discussion concentrates on mechanisms by which $\mathrm{A} \beta$ can drive vascular pathology. The reverse scenario is also a possibility, i.e., that vascular pathology could accelerate AD.

$$
\mathrm{AD} \rightleftarrows \text { Vascular Dysfunction }
$$

Clearance of $A \beta$ is critical to keep its concentration low in the brain and the cerebrovascular circulation, and decreased clearance may be a major cause of increased $\mathrm{A} \beta$ deposition in the $\mathrm{AD}$ brain $(109,110)$. Mechanisms that remove $A \beta$ from the brain include $\mathrm{BBB}$ transport and movement from the CSF and parenchyma into the blood (111-114). If the cerebrovascular system were compromised, it could impede removal of $A \beta$ and lead to increased concentration in the brain. Thus, one could envisage a vicious cycle whereby $A \beta$ negatively affects the circulation, which in turn reduces clearance of $A \beta$ and increases its toxic effects.

Another possible contribution of vascular pathology leading to $\mathrm{AD}$ could be $\mathrm{BBB}$ breakdown. Patients with mild cognitive impairment have been shown to have a compromised BBB (115). This condition could allow plasma proteins, including fibrinogen, to gain access to the brain parenchyma (116) where they could contribute to inflammation and promote neurodegeneration.

Finally, decreases in blood flow can lead to hypoxic tissues and the induction of the hypoxia-inducible factor HIF-1 $\alpha$. This transcription factor can activate $\gamma$-secretase, which could lead to increased $A \beta$ production $(117,118)$. Thus, one can envisage a vicious cycle whereby $A \beta$ causes vascular insufficiency, which in turn leads to increased $A \beta$.

\section{Diagnostic possibilities stemming from vascular contributions to $A D$}

As mentioned, if vascular dysregulation is a significant factor in some $\mathrm{AD}$ cases, it opens a therapeutic window to treat one aspect of the pathology. In addition, vascular involvement offers a possibility of blood-based biomarkers that could help identify contributing pathologies. Analysis of plasma has shown that AD patients and $\mathrm{AD}$ mice have increased contact system activation, as evidenced by increased FXII activation and HK cleavage $(99,100)$. To accurately assess contact activation in blood requires careful attention to many variables, including blood collection, anticoag- ulation, plasma preparation, sample storage, and analytical procedures (119). Nevertheless, it is possible to standardize procedures to maximize reproducibility. Having a biomarker in blood as an early diagnostic to help guide treatment could lead to a significant benefit for some patients.

\section{Cenetics of vascular involvement in $A D$}

This review has concentrated on vascular and inflammatory drivers of AD. In genetic studies, inflammatory genes have emerged as risk factors for $\mathrm{AD}(120,121)$. If vascular dysfunction is also a contributing factor in $\mathrm{AD}$ pathogenesis, why haven't genes associated with clotting and hemostasis turned up in screens for risk factors? One possible reason is that genes that inhibit clotting might prevent or delay $\mathrm{AD}$, so they would not be observed in significant numbers in $\mathrm{AD}$ populations, but rather, in individuals at low risk for $\mathrm{AD}$. One would need to look for protective genes; such a study has been done, and it uncovered an APP mutation that confers protection against $\mathrm{AD}$ (122). However, mutations that induce changes in clotting may also carry a risk of bleeding, so these patients might be present in low numbers in the aged population. It is clear that several genes identified as risk factors for AD, including Apo E4 (123) and PICALM (124, $125)$, can affect cerebrovascular function via the BBB $(126,127)$.

\section{Conclusions}

Multiple contributing pathologies affect the risk of developing AD. Just as treating multiple pathways in cancer has improved outcomes, a similar evolution of therapy can be envisaged for AD. The development of assays to classify patients and treat their specific constellation of pathologies will be required to make progress in treatment. Although reversal of cognitive decline would be the ideal treatment, and next to that a complete inhibition of progression, simply slowing the rate of progression of symptoms by $50 \%$ would be a very significant advance. Accomplishing this goal is going to require, in general, a wider range of analysis and treatment options than are currently employed, and specifically, more thorough investigations into correlations between $\mathrm{AD}$ and vascular dysfunction.

\section{Acknowledgments}

I am very grateful to Marta Cortes-Canteli, Erin H. Norris, Carol C. Strickland, Harold Varmus, and Daria Zamolodchikov for comments on the manuscript. I thank the following for generous support of the work from our laboratory: NIH NS 50537; Robertson Therapeutic Discovery Institute; Alzheimer's Drug Discovery, Rudin Family, Mellam Family, and Mary \& James G. Wallach Foundations; Cure Alzheimer's Fund; Louis Herlands; John A. Herrmann Jr.

Address correspondence to: Sidney Strickland, Laboratory of Neurobiology and Genetics, 1230 York Avenue, New York, New York 10021, USA. Phone: 212.327.8705; Email: strickland@rockefeller.edu.
1. Jack CR Jr, et al. Tracking pathophysiological processes in Alzheimer's disease: an updated hypothetical model of dynamic biomarkers. Lancet Neurol. 2013;12(2):207-216.

2. Nixon RA, Yang DS. Autophagy failure in Alzheimer's disease - locating the primary defect. Neurobiol Dis. 2011;43(1):38-45.
3. Tu S, Okamoto S, Lipton SA, Xu H. Oligomeric $\mathrm{A} \beta$-induced synaptic dysfunction in Alzheimer's disease. Mol Neurodegener. 2014;9:48.

4. Mamelak M. Energy and the Alzheimer brain. Neurosci Biobehav Rev. 2017;75:297-313.

5. Wyss-Coray T, Rogers J. Inflammation in Alzheimer disease-a brief review of the basic science and clinical literature. Cold Spring Harb Perspect Med. 2012;2(1):a006346.

6. Iadecola C. The pathobiology of vascular dementia. Neuron. 2013;80(4):844-866.

7. Zlokovic BV. Neurovascular pathways to neurodegeneration in Alzheimer's disease and other disorders. Nat Rev Neurosci. 2011;12(12):723-738. 
8. Serrano-Pozo A, Frosch MP, Masliah E, Hyman BT. Neuropathological alterations in Alzheimer disease. Cold Spring Harb Perspect Med. 2011;1(1):a006189.

9. Yamada M. Cerebral amyloid angiopathy: emerging concepts. J Stroke. 2015;17(1):17-30.

10. Farkas E, Luiten PG. Cerebral microvascular pathology in aging and Alzheimer's disease. Prog Neurobiol. 2001;64(6):575-611.

11. Kalaria RN. Small vessel disease and Alzheimer's dementia: pathological considerations. Cerebrovasc Dis. 2002;13(suppl 2):48-52.

12. Humpel C. Chronic mild cerebrovascular dysfunction as a cause for Alzheimer's disease? Exp Gerontol. 2011;46(4):225-232.

13. Kisler K, Nelson AR, Montagne A, Zlokovic BV. Cerebral blood flow regulation and neurovascular dysfunction in Alzheimer disease. Nat Rev Neurosci. 2017;18(7):419-434.

14. Snyder HM, et al. Vascular contributions to cognitive impairment and dementia including Alzheimer's disease. Alzheimers Dement. 2015;11(6):710-717.

15. Vinters HV. Emerging concepts in Alzheimer's disease. Annu Rev Pathol. 2015;10:291-319.

16. Serrano-Pozo A, Qian J, Monsell SE, Frosch MP, Betensky RA, Hyman BT. Examination of the clinicopathologic continuum of Alzheimer disease in the autopsy cohort of the National Alzheimer Coordinating Center. J Neuropathol Exp Neurol. 2013;72(12):1182-1192.

17. Toledo JB, et al. Contribution of cerebrovascular disease in autopsy confirmed neurodegenerative disease cases in the National Alzheimer's Coordinating Centre. Brain. 2013;136(pt 9):2697-2706.

18. Arvanitakis Z, Capuano AW, Leurgans SE, Bennett DA, Schneider JA. Relation of cerebral vessel disease to Alzheimer's disease dementia and cognitive function in elderly people: a cross-sectional study. Lancet Neurol. 2016;15(9):934-943.

19. Morris JK, et al. Aerobic exercise for Alzheimer's disease: a randomized controlled pilot trial. PLoS One. 2017;12(2):e0170547.

20. Lautenschlager NT, et al. Effect of physical activity on cognitive function in older adults at risk for Alzheimer disease: a randomized trial. JAMA. 2008;300(9):1027-1037.

21. Erickson KI, et al. Exercise training increases size of hippocampus and improves memory. Proc Natl Acad Sci U S A. 2011;108(7):3017-3022.

22. Ruscheweyh R, et al. Physical activity and memory functions: an interventional study. Neurobiol Aging. 2011;32(7):1304-1319.

23. Liu R, et al. Cardiorespiratory fitness as a predictor of dementia mortality in men and women. Med Sci Sports Exerc. 2012;44(2):253-259.

24. Elwood P, et al. Healthy lifestyles reduce the incidence of chronic diseases and dementia: evidence from the Caerphilly cohort study. PLoS One. 2013;8(12):e81877.

25. Buchman AS, Boyle PA, Yu L, Shah RC, Wilson RS, Bennett DA. Total daily physical activity and the risk of $\mathrm{AD}$ and cognitive decline in older adults. Neurology. 2012;78(17):1323-1329.

26. Snowden M, et al. Effect of exercise on cognitive performance in community-dwelling older adults: review of intervention trials and recommendations for public health practice and research. JAm Geriatr Soc. 2011;59(4):704-716.

27. Sink KM, et al. Effect of a 24-month physical activity intervention vs health education on cognitive outcomes in sedentary older adults: The LIFE Randomized Trial. JAMA. 2015;314(8):781-790.

28. Reijmer YD, van Veluw SJ, Greenberg SM. Ischemic brain injury in cerebral amyloid angiopathy. JCereb Blood Flow Metab. 2016;36(1):40-54.

29. Kalaria RN, Akinyemi R, Ihara M. Does vascular pathology contribute to Alzheimer changes? J Neurol Sci. 2012;322(1-2):141-147.

30. de la Torre JC. Is Alzheimer's disease a neurodegenerative or a vascular disorder? Data, dogma, and dialectics. Lancet Neurol. 2004;3(3):184-190.

31. Iturria-Medina Y, Sotero RC, Toussaint PJ, Mateos-Pérez JM, Evans AC, Alzheimer's Disease Neuroimaging Initiative. Early role of vascular dysregulation on late-onset Alzheimer's disease based on multifactorial data-driven analysis. Nat Commun. 2016;7:11934.

32. Lee $\mathrm{S}$, et al. White matter hyperintensities are a core feature of Alzheimer's disease: evidence from the dominantly inherited Alzheimer network. Ann Neurol. 2016;79(6):929-939.

33. Bertram L, Lill CM, Tanzi RE. The genetics of Alzheimer disease: back to the future. Neuron. 2010;68(2):270-281.

34. Shim YS, et al. Pathological correlates of white matter hyperintensities on magnetic resonance imaging. Dement Geriatr Cogn Disord. 2015;39(1-2):92-104.

35. Erten-Lyons D, et al. Neuropathologic basis of white matter hyperintensity accumulation with advanced age. Neurology. 2013;81(11):977-983.

36. Wardlaw JM, Valdés Hernández MC, MuñozManiega S. What are white matter hyperintensities made of? Relevance to vascular cognitive impairment. J Am Heart Assoc. 2015;4(6):001140.

37. Pantoni L. Cerebral small vessel disease: from pathogenesis and clinical characteristics to therapeutic challenges. Lancet Neurol. 2010;9(7):689-701.

38. Wardlaw JM, Smith C, Dichgans M. Mechanisms of sporadic cerebral small vessel disease: insights from neuroimaging. Lancet Neurol. 2013;12(5):483-497.

39. Lindemer ER, et al. White matter signal abnormality quality differentiates mild cognitive impairment that converts to Alzheimer's disease from nonconverters. Neurobiol Aging. 2015;36(9):2447-2457.

40. Brickman AM, et al. Regional white matter hyperintensity volume, not hippocampal atrophy, predicts incident Alzheimer disease in the community. Arch Neurol. 2012;69(12):1621-1627.

41. Brickman AM. Contemplating Alzheimer's disease and the contribution of white matter hyperintensities. Curr Neurol Neurosci Rep. 2013;13(12):415.

42. Grimmer T, et al. White matter hyperintensities predict amyloid increase in Alzheimer's disease. Neurobiol Aging. 2012;33(12):2766-2773.

43. Zhou Y, Yu F, Duong TQ, Alzheimer's Disease Neuroimaging Initiative. White matter lesion load is associated with resting state functional MRI activity and amyloid PET but not FDG in mild cognitive impairment and early Alzheimer's disease patients. JMagn Reson Imaging. 2015;41(1):102-109.
44. Brickman AM, et al. Cerebral autoregulation, beta amyloid, and white matter hyperintensities are interrelated. Neurosci Lett. 2015;592:54-58.

45. Vemuri $P$, et al. Vascular and amyloid pathologies are independent predictors of cognitive decline in normal elderly. Brain. 2015;138(pt 3):761-771.

46. Natté R, Maat-Schieman ML, Haan J, Bornebroek M, Roos RA, van Duinen SG. Dementia in hereditary cerebral hemorrhage with amyloidosis-Dutch type is associated with cerebral amyloid angiopathy but is independent of plaques and neurofibrillary tangles. Ann Neurol. 2001;50(6):765-772.

47. Tomidokoro Y, et al. Iowa variant of familial Alzheimer's disease: accumulation of posttranslationally modified AbetaD23N in parenchymal and cerebrovascular amyloid deposits. Am J Pathol. 2010;176(4):1841-1854.

48. Lleó A, Berezovska O, Growdon JH, Hyman BT. Clinical, pathological, and biochemical spectrum of Alzheimer disease associated with PS-1 mutations. Am J Geriatr Psychiatry. 2004;12(2):146-156.

49. Kattula S, Byrnes JR, Wolberg AS. Fibrinogen and fibrin in hemostasis and thrombosis. Arterioscler Thromb Vasc Biol. 2017;37(3):e13-e21.

50. Davalos D, Akassoglou K. Fibrinogen as a key regulator of inflammation in disease. Semin Immunopathol. 2012;34(1):43-62.

51. Litvinov RI, Weisel JW. What is the biological and clinical relevance of fibrin? Semin Thromb Hemost. 2016;42(4):333-343.

52. Bardehle S, Rafalski VA, Akassoglou K. Breaking boundaries-coagulation and fibrinolysis at the neurovascular interface. Front Cell Neurosci. 2015;9:354

53. Cortes-Canteli M, et al. Fibrinogen and betaamyloid association alters thrombosis and fibrinolysis: a possible contributing factor to Alzheimer's disease. Neuron. 2010;66(5):695-709.

54. Fiala M, et al. Cyclooxygenase-2-positive macrophages infiltrate the Alzheimer's disease brain and damage the blood-brain barrier. Eur J Clin Invest. 2002;32(5):360-371.

55. Cullen KM, Kócsi Z, Stone J. Microvascular pathology in the aging human brain: evidence that senile plaques are sites of microhaemorrhages. Neurobiol Aging. 2006;27(12):1786-1796.

56. Lipinski B, Sajdel-Sulkowska EM. New insight into Alzheimer disease: demonstration of fibrin(ogen)-serum albumin insoluble deposits in brain tissue. Alzheimer Dis Assoc Disord. 2006;20(4):323-326.

57. Ryu JK, McLarnon JG. A leaky blood-brain barrier, fibrinogen infiltration and microglial reactivity in inflamed Alzheimer's disease brain. JCell Mol Med. 2009;13(9A):2911-2925.

58. Viggars AP, et al. Alterations in the blood brain barrier in ageing cerebral cortex in relationship to Alzheimer-type pathology: a study in the MRCCFAS population neuropathology cohort. Neurosci Lett. 2011;505(1):25-30.

59. Hultman K, Cortes-Canteli M, Bounoutas A, Richards AT, Strickland S, Norris EH. Plasmin deficiency leads to fibrin accumulation and a compromised inflammatory response in the mouse brain. J Thromb Haemost. 2014;12(5):701-712.

60. Paul J, Strickland S, Melchor JP. Fibrin deposition accelerates neurovascular damage and neuroinflammation in mouse models of Alzheimer's 
disease. J Exp Med. 2007;204(8):1999-2008.

61. Cortes-Canteli M, Mattei L, Richards AT, Norris $\mathrm{EH}$, Strickland S. Fibrin deposited in the Alzheimer's disease brain promotes neuronal degeneration. Neurobiol Aging. 2015;36(2):608-617.

62. Barrachina M, Maes T, Buesa C, Ferrer I. Lysosome-associated membrane protein 1 (LAMP-1) in Alzheimer's disease. Neuropathol Appl Neurobiol. 2006;32(5):505-516.

63. Armstrong A, et al. Lysosomal network proteins as potential novel CSF biomarkers for Alzheimer's disease. Neuromolecular Med. 2014;16(1):150-160.

64. Condello C, Schain A, Grutzendler J. Multicolor time-stamp reveals the dynamics and toxicity of amyloid deposition. Sci Rep. 2011;1:19.

65. Hashimoto T, Ogino K, Shin RW, Kitamoto T, Kikuchi T, Shimizu N. Age-dependent increase in lysosome-associated membrane protein 1 and early-onset behavioral deficits in APPSL transgenic mouse model of Alzheimer's disease. Neurosci Lett. 2010;469(2):273-277.

66. Pérez-Gracia E, Torrejón-Escribano B, Ferrer I. Dystrophic neurites of senile plaques in Alzheimer's disease are deficient in cytochrome c oxidase. Acta Neuropathol. 2008;116(3):261-268.

67. Hultman K, Strickland S, Norris EH. The APOE $\varepsilon 4 / \varepsilon 4$ genotype potentiates vascular fibrin(ogen) deposition in amyloid-laden vessels in the brains of Alzheimer's disease patients. JCereb Blood Flow Metab. 2013;33(8):1251-1258.

68. van Oijen M, Witteman JC, Hofman A, Koudstaal PJ, Breteler MM. Fibrinogen is associated with an increased risk of Alzheimer disease and vascular dementia. Stroke. 2005;36(12):2637-2641.

69. Xu G, Zhang H, Zhang S, Fan X, Liu X. Plasma fibrinogen is associated with cognitive decline and risk for dementia in patients with mild cognitive impairment. Int JClin Pract. 2008;62(7):1070-1075.

70. Craig-Schapiro R, et al. Multiplexed immunoassay panel identifies novel CSF biomarkers for Alzheimer's disease diagnosis and prognosis. PLoS One. 2011;6(4):e18850.

71. Vafadar-Isfahani B, et al. Identification of SPARC-like 1 protein as part of a biomarker panel for Alzheimer's disease in cerebrospinal fluid. JAlzheimers Dis. 2012;28(3):625-636.

72. Lee JW, et al. Fibrinogen gamma-A chain precursor in CSF: a candidate biomarker for Alzheimer's disease. BMC Neurol. 2007;7:14.

73. Thambisetty M, et al. Plasma biomarkers of brain atrophy in Alzheimer's disease. PLoS One. 2011;6(12):e28527.

74. Yang H, Lyutvinskiy Y, Herukka SK, Soininen H, Rutishauser D, Zubarev RA. Prognostic polypeptide blood plasma biomarkers of Alzheimer's disease progression. JAlzheimers Dis. 2014;40(3):659-666.

75. Chiam JT, Dobson RJ, Kiddle SJ, Sattlecker M. Are blood-based protein biomarkers for Alzheimer's disease also involved in other brain disorders? A systematic review. J Alzheimers Dis. 2015;43(1):303-314.

76. Musiek ES, Holtzman DM. Three dimensions of the amyloid hypothesis: time, space and 'wingmen'. Nat Neurosci. 2015;18(6):800-806.

77. Selkoe DJ, Hardy J. The amyloid hypothesis of
Alzheimer's disease at 25 years. EMBO Mol Med. 2016;8(6):595-608

78. Tanzi RE. The genetics of Alzheimer disease. Cold Spring Harb Perspect Med. 2012;2(10):a006296

79. Ahn HJ, Zamolodchikov D, Cortes-Canteli M, Norris EH, Glickman JF, Strickland S. Alzheimer's disease peptide $\beta$-amyloid interacts with fibrinogen and induces its oligomerization. Proc Natl Acad Sci U S A. 2010;107(50):21812-21817.

80. Zamolodchikov D, Strickland S. A $\beta$ delays fibrin clot lysis by altering fibrin structure and attenuating plasminogen binding to fibrin. Blood. 2012;119(14):3342-3351.

81. Barber M, Tait RC, Scott J, Rumley A, Lowe GD, Stott DJ. Dementia in subjects with atrial fibrillation: hemostatic function and the role of anticoagulation. J Thromb Haemost. 2004;2(11):1873-1878.

82. Walsh AC. Anticoagulant therapy for Alzheimer's disease. J Neuropsychiatry Clin Neurosci. 1996;8(3):361-362.

83. Bergamaschini L, et al. Peripheral treatment with enoxaparin, a low molecular weight heparin, reduces plaques and beta-amyloid accumulation in a mouse model of Alzheimer's disease. J Neurosci. 2004;24(17):4181-4186.

84. Timmer NM, van Dijk L, van der Zee CE, Kiliaan A, de Waal RM, Verbeek MM. Enoxaparin treatment administered at both early and late stages of amyloid $\beta$ deposition improves cognition of APPswe/ PS1dE9 mice with differential effects on brain $A \beta$ levels. Neurobiol Dis. 2010;40(1):340-347.

85. Tripathy D, Sanchez A, Yin X, Luo J, Martinez J, Grammas P. Thrombin, a mediator of cerebrovascular inflammation in $\mathrm{AD}$ and hypoxia. Front Aging Neurosci. 2013;5:19.

86. Ryu JK, et al. Blood coagulation protein fibrinogen promotes autoimmunity and demyelination via chemokine release and antigen presentation. Nat Commun. 2015;6:8164.

87. Ahn HJ, et al. A novel A $\beta$-fibrinogen interaction inhibitor rescues altered thrombosis and cognitive decline in Alzheimer's disease mice. J Exp Med. 2014;211(6):1049-1062.

88. Bagyinszky E, Giau VV, Shim K, Suk K, An SSA, Kim S. Role of inflammatory molecules in the Alzheimer's disease progression and diagnosis. J Neurol Sci. 2017;376:242-254.

89. Akiyama H, et al. Inflammation and Alzheimer's disease. Neurobiol Aging. 2000;21(3):383-421.

90. Spangenberg EE, et al. Eliminating microglia in Alzheimer's mice prevents neuronal loss without modulating amyloid- $\beta$ pathology. Brain . 2016;139(pt 4):1265-1281.

91. Asai $\mathrm{H}$, et al. Depletion of microglia and inhibition of exosome synthesis halt tau propagation. Nat Neurosci. 2015;18(11):1584-1593.

92. Renné $\mathrm{T}$. The procoagulant and proinflammatory plasma contact system. Semin Immunopathol. 2012;34(1):31-41.

93. White GC II, Marder VJ, Schulman S, Aird WC, Bennett JS. Overview of basic coagulation and fibrinolysis. In: Marder VJ, Aird WC, Bennett JS, Schulman S, White GC, eds. Hemostasis and thrombosis: basic principles and clinical practice. 6th ed. Philadelphia, Pennsylvania, USA: Wolters Kluwer/Lippincott, Williams \& Wilkins; 2013:103-109.
94. Leeb-Lundberg LM, Marceau F, Müller-Esterl W, Pettibone DJ, Zuraw BL. International union of pharmacology. XLV. Classification of the kinin receptor family: from molecular mechanisms to pathophysiological consequences. Pharmacol Rev. 2005;57(1):27-77.

95. Renné T. The Factor XII-driven plasma contact system. In: Marder VJ, Aird WC, Bennett JS, Schulman S, White GC, eds. Hemostasis and thrombosis: basic principles and clinical practice. 6th ed. Philadelphia, Pennsylvania, USA: Wolters Kluwer/Lippincott, Williams \& Wilkins; 2013:242-253.

96. Yasuhara O, Walker DG, McGeer PL. Hageman factor and its binding sites are present in senile plaques of Alzheimer's disease. Brain Res. 1994;654(2):234-240.

97. Ashby EL, Love S, Kehoe PG. Assessment of activation of the plasma kallikrein-kinin system in frontal and temporal cortex in Alzheimer's disease and vascular dementia. Neurobiol Aging. 2012;33(7):1345-1355.

98. Bergamaschini L, Donarini C, Foddi C, Gobbo G, Parnetti L, Agostoni A. The region 1-11 of Alzheimer amyloid-beta is critical for activation of contact-kinin system. Neurobiol Aging. 2001;22(1):63-69.

99. Zamolodchikov D, Chen ZL, Conti BA, Renné T, Strickland S. Activation of the factor XII-driven contact system in Alzheimer's disease patient and mouse model plasma. Proc Natl Acad Sci US A. 2015;112(13):4068-4073.

100.Chen ZL, Revenko AS, Singh P, MacLeod AR, Norris EH, Strickland S. Depletion of coagulation factor XII ameliorates brain pathology and cognitive impairment in Alzheimer disease mice. Blood. 2017;129(18):2547-2556.

101.Zamolodchikov D, Renné T, Strickland S. The Alzheimer's disease peptide $\beta$-amyloid promotes thrombin generation through activation of coagulation factor XII. J Thromb Haemost. 2016;14(5):995-1007.

102. Yang L, Li Y, Bhattacharya A, Zhang Y. A plasma proteolysis pathway comprising blood coagulation proteases. Oncotarget. 2016;7(27):40919-40938.

103. Shibayama Y, Joseph K, Nakazawa Y, Ghebreihiwet B, Peerschke EI, Kaplan AP. Zinc-dependent activation of the plasma kinin-forming cascade by aggregated $\beta$ amyloid protein. Clin Immunol. 1999;90(1):89-99.

104. Maas C, et al. Misfolded proteins activate factor XII in humans, leading to kallikrein formation without initiating coagulation. JClin Invest. 2008;118(9):3208-3218.

105. Renne T, Schmaier AH, Nickel KF, Blomback $\mathrm{M}$, Maas C. In vivo roles of factor XII. Blood. 2012;120(22):4296-4303.

106.Merkulov S, et al. Deletion of murine kininogen gene 1 (mKng1) causes loss of plasma kininogen and delays thrombosis. Blood. 2008;111(3):1274-1281.

107. Sheffer AL, MacGinnitie AJ, Campion M, Stolz LE, Pullman WE. Outcomes after ecallantide treatment of laryngeal hereditary angioedema attacks. Ann Allergy Asthma Immunol. 2013;110(3):184-188.e2.

108. Chyung Y, et al. A phase 1 study investigating DX-2930 in healthy subjects. Ann Allergy Asthma Immunol. 2014;113(4):460-466.e2. 
109. Mawuenyega KG, et al. Decreased clearance of CNS beta-amyloid in Alzheimer's disease. Science. 2010;330(6012):1774.

110.Iturria-Medina Y, Sotero RC, Toussaint PJ, Evans AC, Alzheimer's Disease Neuroimaging Initiative. Epidemic spreading model to characterize misfolded proteins propagation in aging and associated neurodegenerative disorders. PLOS Comput Biol. 2014;10(11):e1003956.

111. Tarasoff-Conway JM, et al. Clearance systems in the brain-implications for Alzheimer disease. Nat Rev Neurol. 2015;11(8):457-470.

112. Zhao Z, Nelson AR, Betsholtz C, Zlokovic BV. Establishment and dysfunction of the bloodbrain barrier. Cell. 2015;163(5):1064-1078.

113. Shibata M, et al. Clearance of Alzheimer's amyloid-ss(1-40) peptide from brain by LDL receptor-related protein-1 at the blood-brain barrier. J Clin Invest. 2000;106(12):1489-1499.

114. Deane R, et al. LRP/amyloid beta-peptide interaction mediates differential brain efflux of $A \beta$ isoforms. Neuron. 2004;43(3):333-344.

115. Montagne A, et al. Blood-brain barrier breakdown in the aging human hippocampus. Neuron. 2015;85(2):296-302.

116. Deane R, et al. RAGE mediates amyloid-beta peptide transport across the blood-brain bar- rier and accumulation in brain. Nat Med. 2003;9(7):907-913.

117. Gertsik N, Chiu D, Li YM. Complex regulation of $\gamma$-secretase: from obligatory to modulatory subunits. Front Aging Neurosci. 2014;6:342.

118. Villa JC, et al. Nontranscriptional role of Hif-1 $\alpha$ in activation of $\gamma$-secretase and notch signaling in breast cancer. Cell Rep. 2014;8(4):1077-1092.

119. Loeffen R, et al. Preanalytic variables of thrombin generation: towards a standard procedure and validation of the method. J Thromb Haemost. 2012;10(12):2544-2554.

120. Zhang ZG, Li Y, Ng CT, Song YQ. Inflammation in Alzheimer's disease and molecular genetics: recent update. Arch Immunol Ther Exp (Warsz). 2015;63(5):333-344.

121. Sims R, et al. Rare coding variants in PLCG2, $\mathrm{ABI} 3$, and TREM2 implicate microglial-mediated innate immunity in Alzheimer's disease. Nat Genet. 2017;49(9):1373-1384.

122.Jonsson T, et al. A mutation in APP protects against Alzheimer's disease and age-related cognitive decline. Nature. 2012;488(7409):96-99.

123. Strittmatter WJ, et al. Apolipoprotein E: highavidity binding to beta-amyloid and increased frequency of type 4 allele in late-onset familial Alzheimer disease. Proc Natl Acad Sci U S A.
1993;90(5):1977-1981.

124. Harold D, et al. Genome-wide association study identifies variants at CLU and PICALM associated with Alzheimer's disease. Nat Genet. 2009;41(10):1088-1093.

125. Lambert JC, et al. Genome-wide association study identifies variants at CLU and CR1 associated with Alzheimer's disease. Nat Genet. 2009;41(10):1094-1099.

126.Zhao Z, et al. Central role for PICALM in amyloid- $\beta$ blood-brain barrier transcytosis and clearance. Nat Neurosci. 2015;18(7):978-987.

127. Bell RD, et al. Apolipoprotein E controls cerebrovascular integrity via cyclophilin A. Nature. 2012;485(7399):512-516.

128. Atri A, et al. Cumulative, additive benefits of memantine-donepezil combination over component monotherapies in moderate to severe Alzheimer's dementia: a pooled area under the curve analysis. Alzheimers Res Ther. 2015;7(1):28.

129. Hendrix JA, et al. Challenges, solutions, and recommendations for Alzheimer's disease combination therapy. Alzheimers Dement. 2016;12(5):623-630.

130. Schmitt B, Bernhardt T, Moeller HJ, Heuser I, Frölich L. Combination therapy in Alzheimer's disease: a review of current evidence. CNS Drugs. 2004;18(13):827-844. 